\title{
Bilateral choroidal neovascularization associated with optic nerve head drusen treated by antivascular endothelial growth factor therapy
}

This article was published in the following Dove Press journal:

Clinical Ophthalmology

10 February 2012

Number of times this article has been viewed

\author{
Barbara Delas \\ Lorena Almudí \\ Anabel Carreras \\ Mouafk Asaad \\ Ophthalmology Service, Hospital \\ de Terrassa, Barcelona, Spain
}

Correspondence: Barbara Delas Ophthalmology Service, Hospital de Terrassa, Ctra Torrebonica s/n. Terrassa 08227, Barcelona, Spain Tel +34937 310007 Fax +34 937310959

Email barbaradelasalos@gmail.com
Objective: To report a good clinical outcome in a patient with bilateral choroidal neovascularization (CNV) associated with optic nerve head drusen (ONHD) treated with intravitreal ranibizumab injection.

Methods: A 12-year-old girl was referred for loss of right eye vision detected in a routine check-up. Best-corrected visual acuity (BCVA) was hand movements in the right eye and 0.9 in the left eye. Funduscopy revealed the presence of superficial and buried bilateral ONHD, which was confirmed by ultrasonography and computed tomography, and the study was completed with perimetry. The presence of bilateral CNV, active in the right eye, was observed and subsequently confirmed using fluorescein angiography and optical coherence tomography.

Results: Treatment with two consecutive injections of intravitreal ranibizumab resulted in inactivation of the neovascular membrane with subretinal fluid reabsorption and improved right eye BCVA. After 12 months' follow-up, this was 20/60 and stable.

Conclusion: Although there are no published studies of safety in children, antiangiogenic therapy for $\mathrm{CNV}$ secondary to ONHD may be useful and safe. A search of the literature produced only one previously reported case of ONHD-associated CNV treated with antivascular endothelial growth factor alone.

Keywords: optic nerve head drusen, anti-VEGF, children, neovascularisation

\section{Introduction}

Optic nerve head drusen (ONHD) are found in 3.4-24 individuals per 1000 population, bilaterally in $75 \%$ of cases. Most are detected in a routine eye examination without the patient reporting visual loss or other symptoms. ${ }^{1}$ Regarding location, ONHD may be superficial or buried, in which case differential diagnosis with other causes of pseudopapilledema is required. In children, superficial drusen are rare; it is more common to see congestive papillae without excavation due to the accumulation of extracellular material in deeper layers. ${ }^{2}$ With age, drusen increase in size and become more visible, so in adults we observe filled papillae with kidney-shaped edges and irregular glistening white points.

Most patients with ONHD are asymptomatic and diagnosed incidentally in routine funduscopy. Those with symptoms often report insidious visual loss due to visual field alterations, and sudden visual loss is rare. ONHD progressively increase in size with age, becoming more visible due to the accumulation of calcium, which correlates with progression of visual field defects. The natural history of ONHD is variable, and in some cases spontaneous involution has been described. ${ }^{3}$ Although there is currently no effective treatment, correct diagnosis is essential to differentiate it from papilledema, 
in order to avoid unnecessary neurological examinations and especially to rule out neurological pathology compatible with the appearance of pseudopapilledema.

The diagnosis of ONHD is made by funduscopy in most cases. However, additional examinations may be useful to confirm the diagnosis or, in doubtful cases, to detect possible complications.

B-scan ultrasound is particularly useful to detect deep calcium deposits, which appear as highly reflective oval lesions with posterior acoustic shadowing when using medium and low frequencies. ${ }^{4}$ In fact, ultrasound is the tool of choice for the detection of ONHD, although the experience of the sonographer is essential to detect small-sized drusen., ${ }^{5,6}$

The calcium content of ONHD is what makes them appear as bright spots in computed tomography (CT), which is considered a superior method for detecting deep drusen compared with ophthalmoscopy. It also offers the advantage of allowing possible intracranial mass to be ruled out. ${ }^{7}$ However, the amplitude of CT cuts used at the optic nerve head means that small drusen are sometimes not reflected, making it less sensitive than ultrasound for detection. This, added to high cost and difficulty of use, limits the usefulness of CT for initial diagnosis of ONHD.

ONHD are autofluorescent, so they emit a glow when viewed through filters prior to fluorescein injection. This phenomenon is due to the calcified refractile material that composes the drusen. Fundus autofluorescence imaging is more sensitive than ophthalmoscopy for the detection of drusen but less so than ultrasound. ${ }^{8}$

In fluorescein angiography (FA) we observe hyperfluorescence of the optic disk. This technique allows us to differentiate ONHD from other entities such as papilledema or neovascularization, where the initial hyperfluorescence increases during the angiogram due to contrast extravasation. Although FA is technically unnecessary for the diagnosis of ONHD, it is useful for detecting and monitoring some of the complications.

Optical coherence tomography (OCT) provides early evidence of retinal nerve fiber layer thinning, which is associated with an increase in the quantity and/or size of drusen. ${ }^{8}$ Like FA, it is a very useful technique in the management of some vascular complications associated with ONHD.

Despite the generally benign nature of the picture, there may be vascular complications that compromise visual prognosis in these patients, including choroidal neovascularization (CNV), ischemic optic neuritis, central retinal artery occlusion, central retinal vein occlusion, and retinal or optic nerve hemorrhage. Among the possible mechanisms involved in these complications is the compressive effect of drusen on surrounding blood vessels, causing mechanical damage of vascular integrity, vascular congestion, or ischemia.

CNV associated with ONHD has been described but remains a very rare complication. In the few existing published cases, CNV was treated with laser photocoagulation, photodynamic therapy, or macular surgery. To our knowledge, the literature contains only one case where CNV secondary to ONHD was treated with antivascular endothelial growth factor (anti-VEGF) alone, ${ }^{9}$ and another case with intravitreal ranibizumab (IVR) injection combined with laser photocoagulation. ${ }^{10}$ This combined strategy yielded good results in both cases but does not allow any conclusions on the therapeutic utility of IVR alone. We report the second case of treatment with IVR alone for CNV secondary to ONHD, with no adverse effects and good response.

\section{Case report}

A 12-year-old girl was referred to the ophthalmology department at Terrassa Hospital, Barcelona, Spain, by a private optician who, in a routine eye examination, found right eye best-corrected visual acuity (BCVA) of counting fingers at $20 \mathrm{~cm}$ and 20/20 in the left eye. The patient reported no loss of vision or other associated symptoms and had no personal or family history of interest. Intraocular pressure and anterior segment examination were normal. Funduscopy revealed the presence of bilateral superficial ONHD, marked venous congestion and arterial stenosis, the presence of fibrous $\mathrm{CNV}$ in the papillomacular bundle in both eyes, and serous detachment of the retina in the right eye reaching the macular area (Figures 1 and 2). B-scan ultrasound (Figure 3) and CT

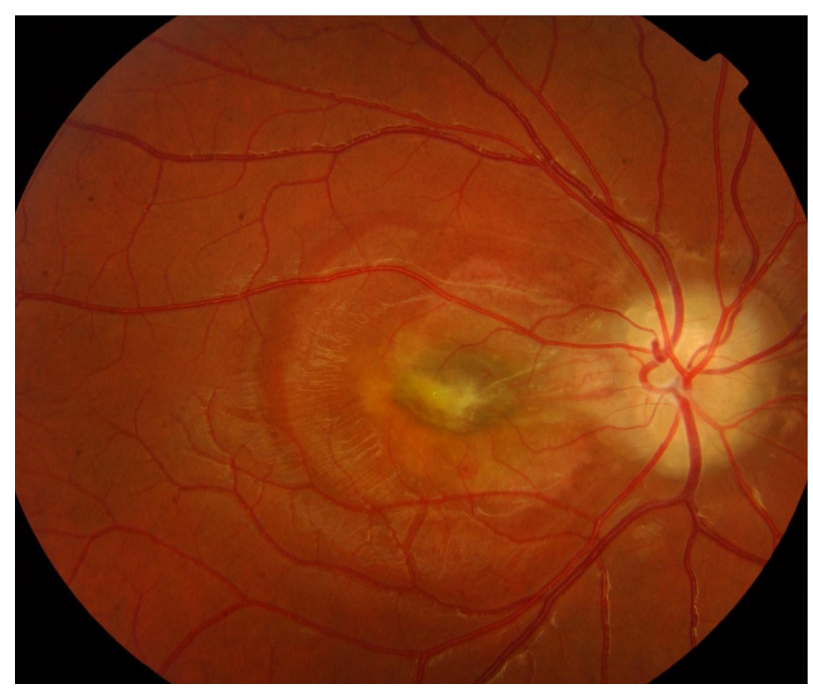

Figure I Optic nerve head drusen associated with an active choroidal neovascularization in the macular area on the right eye. 


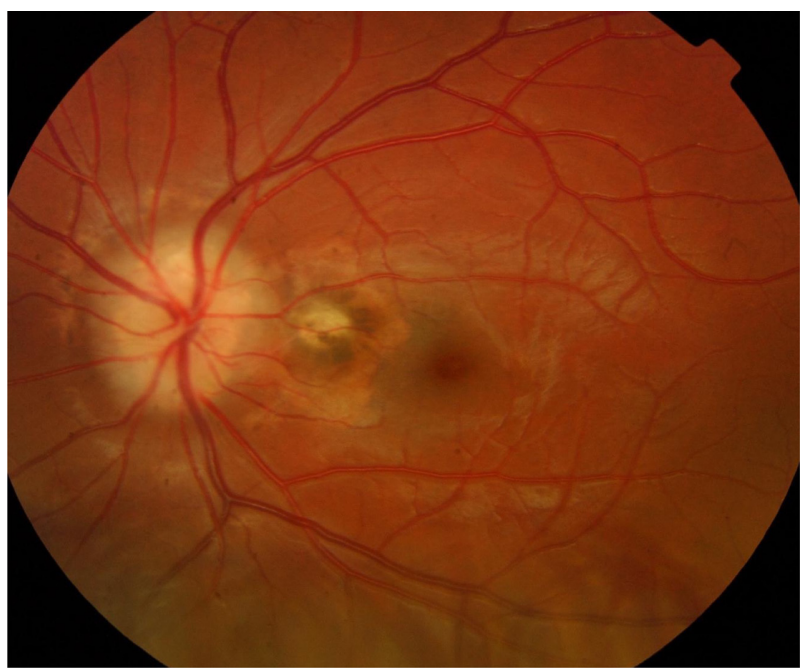

Figure 2 Fibrous choroidal neovascularization in the papillomacular bundle without activity on the left eye.

(Figure 4) confirmed the suspected diagnosis of bilateral ONHD, and FA revealed the presence of active extrafoveal $\mathrm{CNV}$ in the papillomacular bundle of the right eye and bilateral pigment epithelial atrophy in the juxtapapillary area temporal to the optic nerve (Figures 5 and 6). OCT revealed the presence of a juxtafoveal CNV in both eyes with associated subretinal fluid in the right eye (Figure 7) and a foveal thickness of $318 \mu \mathrm{m}$. The visual field was not evaluated in the right eye due to lack of fixation, and deep, extensive scotomas were observed in the left eye (Figure 8).

With a diagnosis of choroidal neovascular membrane (NVM) secondary to optic nerve drusen, we obtained informed consent to administer treatment with IVR
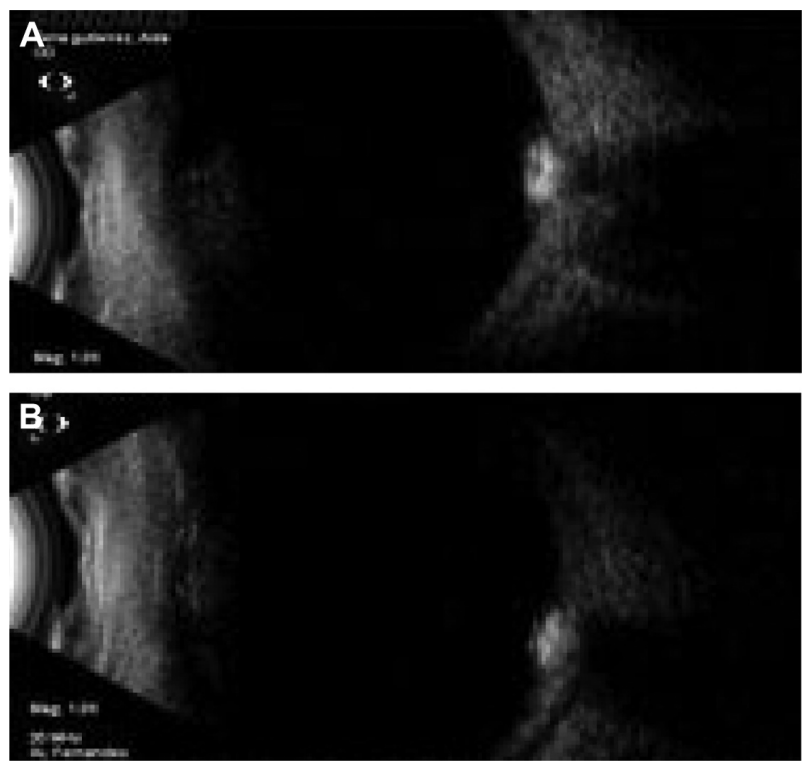

Figure 3 Ultrasound of $(\mathbf{A})$ right eye and $(\mathbf{B})$ left eye.

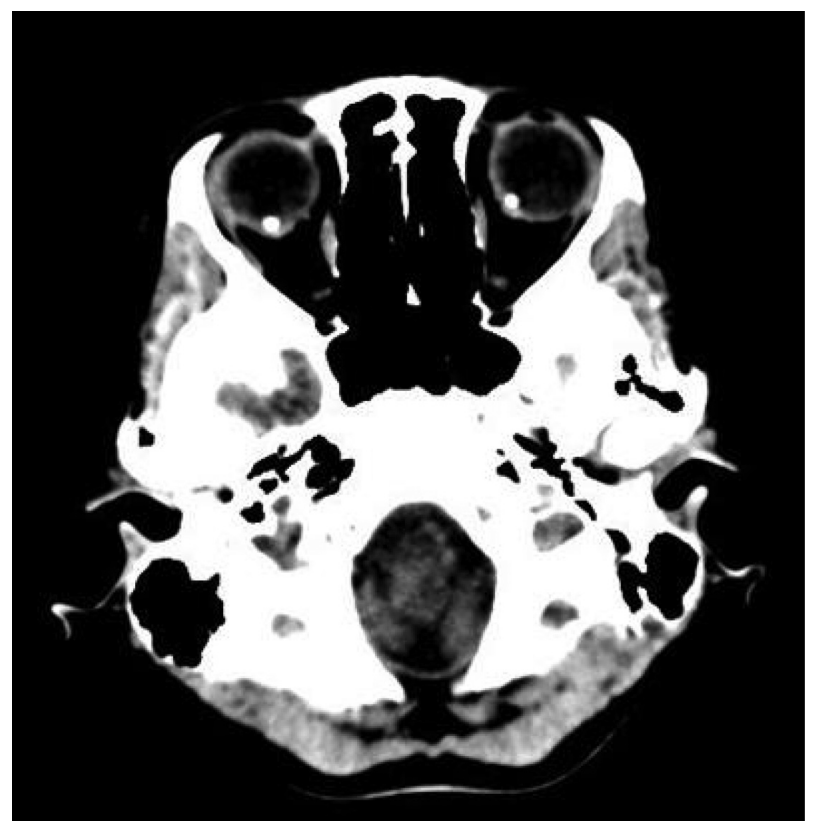

Figure 4 Computed tomography.

injection, given at a dose of $0.5 \mathrm{mg}(0.05 \mathrm{~mL})$ with a 30 -gauge needle $4 \mathrm{~mm}$ from the limbus, under sedation, and after application of topical anesthesia with $2 \%$ lidocaine, under strict asepsis. The patient was treated with topical quinolone three times a day for a week after intravitreal injection. The next day, and 1 week later, intraocular pressure (IOP) was measured and slit-lamp examination and funduscopy were performed. One month after the injection, IOP was normal, visual acuity was two Early Treatment Diabetic Retinopathy Study letters at 4 meters, fundus examination revealed no changes from previous visits, and OCT showed persistence of minimal subretinal fluid, so a second injection of IVR was administered. After the

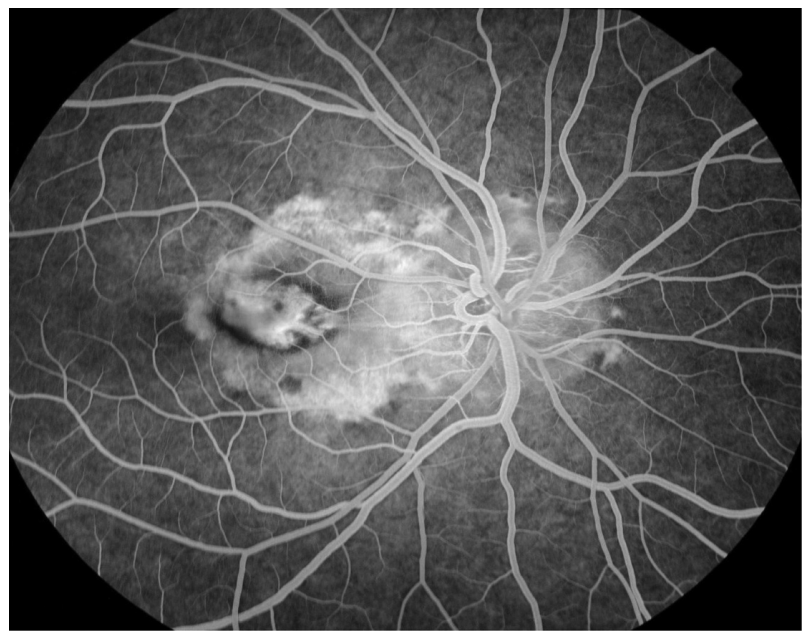

Figure 5 Initial fluorescein angiography. 


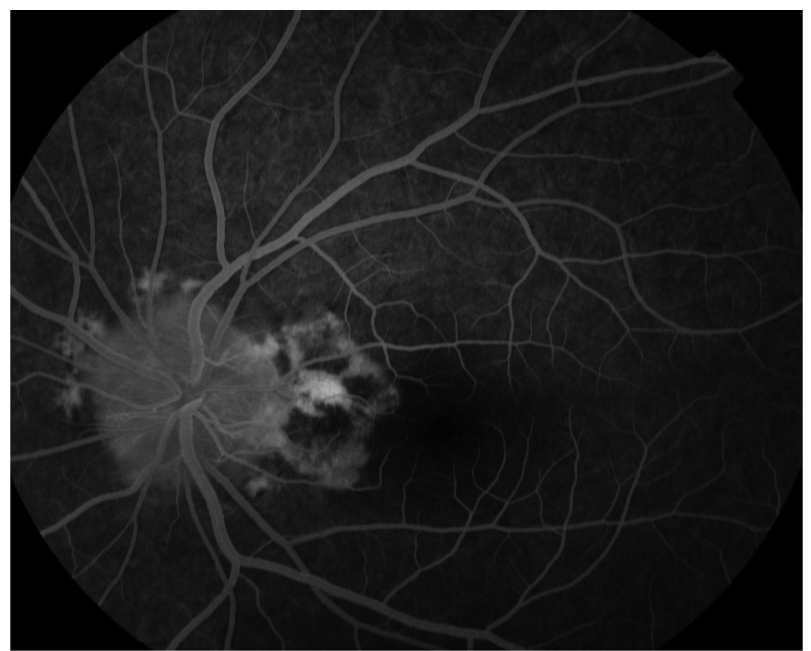

Figure 6 Fluorescein angiography.

second injection the same pattern of visits was followed. At 4 weeks after the second injection, visual acuity was 20/60. Fundus examination showed the NVM without fluid, confirmed by OCT, which also showed a foveal thickness of $295 \mu \mathrm{m}$. From then on, successive visits were made every 6 weeks, with BCVA, IOP, anterior and posterior segment examination, and OCT performed and found to remain stable at every visit. At the end of follow-up, 12 months after the first visit, OCT showed no subretinal fluid and right eye BCVA remained stable at 20/60. The left eye showed no signs of activity at any time. The patient
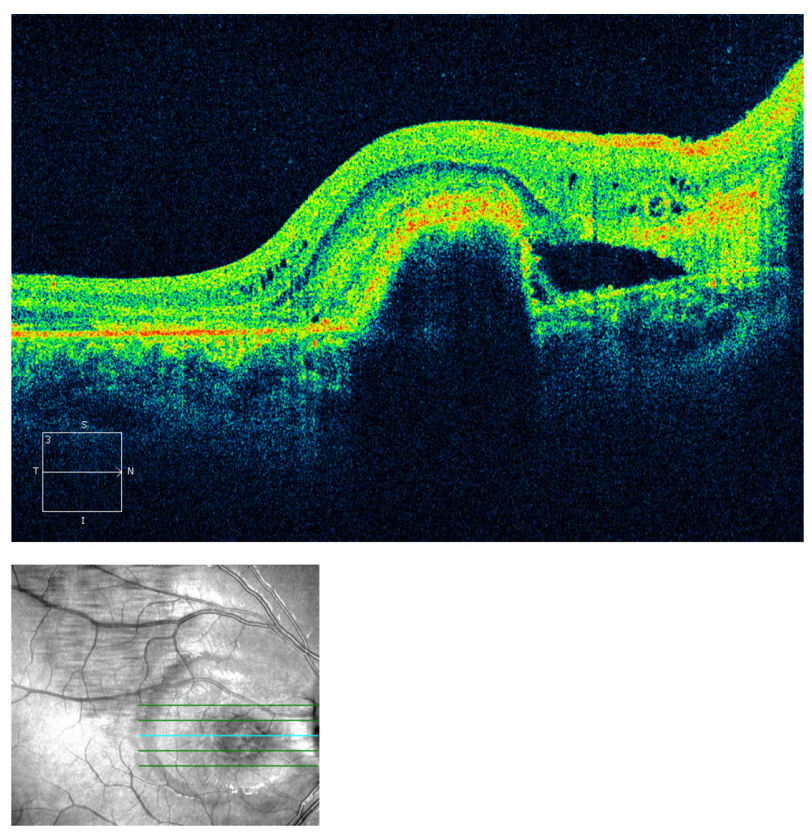

Figure 7 Optical coherence tomography of the right eye. receives regular monitoring for the early detection of possible complications.

\section{Discussion}

The prevalence of ONHD is very low and their association with CNV is extremely rare, with no established treatment regimen. The natural evolution of $\mathrm{CNV}$ associated with ONHD is highly variable, and even spontaneous involution has been described. The macula may be affected early in the progression of the lesion, either by direct extension of the NVM or by associated serous or hemorrhagic retinal detachment, severely compromising visual prognosis.

Over the years, many treatment models have been proposed. Laser photocoagulation was for many years the most widely used treatment for this type of NVM, ${ }^{11}$ although the residual scotoma it produced meant that it was contraindicated in NVM with subfoveal location. Macular surgery also showed good results, although the added surgical risk in these cases required consideration. ${ }^{12}$

Some investigators have advocated the use of photodynamic therapy with verteporfin as safe and effective for the treatment of $\mathrm{CNV}$ associated with symptomatic ONHD. ${ }^{13-15}$

Antiangiogenic therapy is currently the treatment of choice for the treatment of CNV secondary to age-related macular degeneration (AMD). It is also indicated for the treatment of CNV associated with entities such as high myopia, ${ }^{16,17}$ macular edema associated with vascular occlusion ${ }^{18}$ or diabetes mellitus, ${ }^{19}$ and premature retinopathy of prematurity. ${ }^{20}$

\section{Conclusion}

With the introduction of anti-VEGF therapy into daily practice in recent years, the prognosis of retinal disease has changed dramatically, to the point where this therapy has succeeded in changing the course of the disease in some cases. This is most evident in AMD, but anti-VEGF for other entities such as vascular occlusion or diabetic retinopathy has also been extensively studied, with good results.

CNV secondary to ONHD is highly unusual, and widely varying treatment options have been used over the years. Although there are no recommendations in this regard, the results obtained in our patient indicate that antiangiogenic therapy can be useful and safe for the treatment of CNV secondary to optic disk drusen. Larger series are needed to confirm this. The favorable response reported here suggests a further example of the utility of IVR to be added to its known 


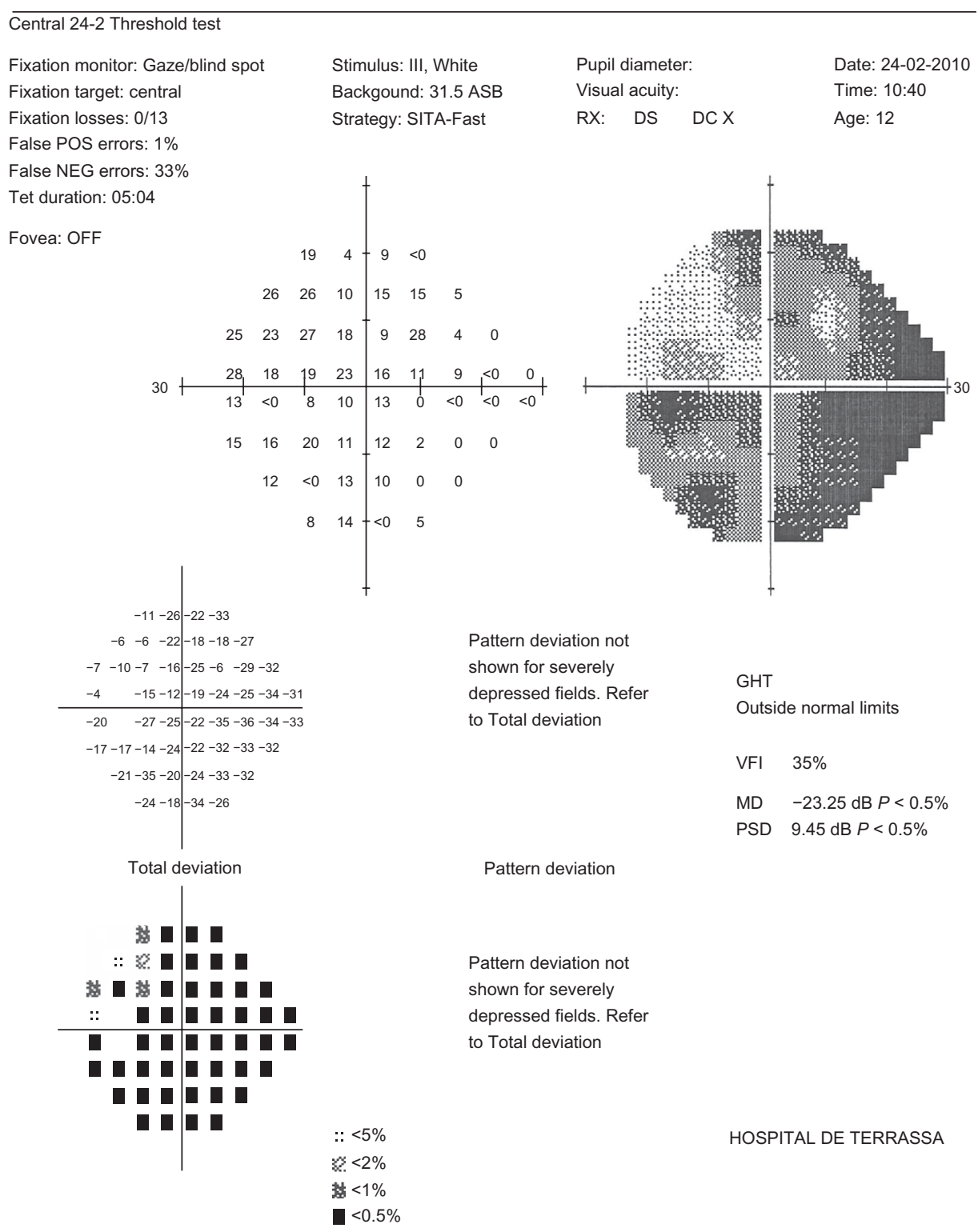

Figure 8 Visual field of the left eye.

beneficial effects on NVM associated with other entities such as AMD and high myopia.

\section{Disclosure}

The authors report no conflict of interest in this work.

\section{References}

1. Auw-Haedrich C, Staubach F, Witschel H. Optic disk drusen. Surv Ophthalmol. 2002;47:515-532.

2. Erkkila H. Optic disc drusen in children. Acta Ophthalmol. 1977; 129(Suppl):3-44.

3. Silvestri G, Archer DB, Johnston PB. Peripapillary subretinal neovascular membranes: the natural history. Eye. 1993;7:398-402.

4. Atta HR. Imaging of the optic nerve with standardised echography. Eye. 1988;2:358-366.

5. Kiegler HR. Comparison of functional findings with results of standardized echography of the optic nerve in optic disk drusen. Wien Klin. 1995;107:651-653.
6. Kurz-Levin MM, Landau K. A comparison of imaging techniques for diagnosing drusen of the optic nerve head. Arch Ophthalmol. 1999;117: 1045-1049.

7. Mullie MA, Sanders MD. Computed tomographic diagnosis of buried drusen of the optic nerve head. Can J Ophthalmol. 1985;20: 114-117.

8. Lam B, Morais C, Pasol J. Drusen of the optic disc. Curr Neurol Neurosci Rep. 2008;8:404-408.

9. Gregory-Evans K, Rai P, Patterson J. Successful treatment of subretinal neovascularization with intravitreal ranibizumab in a child with optic nerve head drusen. J Pediatr Ophthalmol Strabismus. 2009:1-4.

10. Knape RM, Zavaleta EM, Clark CL, et al. Intravitreal bevacizumab treatment of bilateral peripapillary choroidal neovascularization from optic nerve head drusen. JAAPOS. 2011;15:87-90.

11. Delyfer M, Rougier M, Fourmaux E, et al. Laser photocoagulation for choroidal neovascular membrane associated with optic disc drusen. Acta Ophthalmol Scand. 2004;82:236-238.

12. Adan A, Corcóstegui B. Surgical removal of peripapillary choroidal neovascularization associated with optic nerve drusen. Retina. 2004; 24:739-745. 
13. Chaudhry N, Lavaque A, Shah A, Liggett P. Photodynamic therapy for choroidal neovascular membrane secondary to optic nerve drusen. Ophthalmic Surg Lasers Imaging. 2005;36:70-72.

14. Torrent R, Loureiro R, Travassos A, Rui Faria de Abreu J. Bilateral CNV associated with optic nerve drusen treated with photodynamic therapy with verteporfin. Eur J Ophthalmol. 2004;14:434- 437.

15. Silva R, Torrent T, Loureiro R, et al. Bilateral CNV associated with optic nerve drusen treated with photodynamic therapy with verteporfin. Eur J Ophthalmol. 2004;14:434-437.

16. Chan WM, Ohji M, Lai TYY, et al. Choroidal neovascularisation in pathological myopia: an update in management. Br J Ophthalmol. 2005;89:1522-1528.
17. Yamamoto I, Rogers AH, Reichel E, et al. Intravitreal bevacizumab (Avastin) as treatment of subfoveal choroidal neovascularisation secondary to pathological myopia. Br J Opthalmol. 2007;91:157-160.

18. Ahmadi A, Chuo J, Banashkevich A, et al. The effects of intravitreal bevacizumab on patients with macular edema secondary to branch retinal vein occlusion. Can J Ophthalmol. 2009;44:154-159.

19. Parravano MC, Menchini F, Virgili G. Tratamiento antiangiogénico con tipos de factor de crecimiento endotelial antivascular para el edema macular diabético. La Biblioteca Cochrane plus 2010 num 1.

20. Mintz-Hittner HA, Kuffel RR Jr. Intravitreal injection of bevacizumab $\left(\right.$ Avastin ${ }^{\circledR}$ ) for treatment of stage 3 retinopathy of prematurity in zone I or posterior zone I. Retina. 2008;28:831-838.
Clinical Ophthalmology

\section{Publish your work in this journal}

Clinical Ophthalmology is an international, peer-reviewed journal covering all subspecialties within ophthalmology. Key topics include: Optometry; Visual science; Pharmacology and drug therapy in eye diseases; Basic Sciences; Primary and Secondary eye care; Patient Safety and Quality of Care Improvements. This journal is indexed on

\footnotetext{
Submit your manuscript here: http://www.dovepress.com/clinical-ophthalmology-journal
}

\section{Dovepress}

PubMed Central and CAS, and is the official journal of The Society of Clinical Ophthalmology (SCO). The manuscript management system is completely online and includes a very quick and fair peer-review system, which is all easy to use. Visit http://www.dovepress.com/ testimonials.php to read real quotes from published authors. 